Case Report

\title{
Huge Intrathoracic Malignant Peripheral Nerve Sheath Tumor in an Adolescent with Neurofibromatosis Type 1
}

\author{
Jong Hyung Yoon, ${ }^{1}$ Hyun-Sung Lee, ${ }^{1,2}$ Jong In Chun, ${ }^{1}$ Seog-Yun Park, ${ }^{1,3}$ \\ Hyeon Jin Park, ${ }^{1}$ and Byung-Kiu Park ${ }^{1}$ \\ ${ }^{1}$ Center for Pediatric Oncology, National Cancer Center, 323 Ilsan-ro, Ilsandong-gu, Goyang-si, \\ Gyeonggi-do 410-769, Republic of Korea \\ ${ }^{2}$ Center for Lung Cancer, National Cancer Center, 323 Ilsan-ro, Ilsandong-gu, Goyang-si, Gyeonggi-do 410-769, Republic of Korea \\ ${ }^{3}$ Department of Pathology, National Cancer Center, 323 Ilsan-ro, Ilsandong-gu, Goyang-si, Gyeonggi-do 410-769, Republic of Korea
}

Correspondence should be addressed to Byung-Kiu Park; bkpark@ncc.re.kr

Received 12 March 2014; Revised 12 May 2014; Accepted 12 May 2014; Published 25 May 2014

Academic Editor: Maria Moschovi

Copyright (C) 2014 Jong Hyung Yoon et al. This is an open access article distributed under the Creative Commons Attribution License, which permits unrestricted use, distribution, and reproduction in any medium, provided the original work is properly cited.

Malignant peripheral nerve sheath tumor (MPNST) is a rare soft tissue malignancy usually found in patients with neurofibromatosis type 1 (NF1) with a poor outcome. Although MPNST can be found in any part of the body including head and neck or extremities, intrathoracic MPNST with or without NF1 is uncommon, especially in children or adolescents. Reported herein is a case of huge intrathoracic MPNST in a 16-year-old girl with NF1, and a brief review of the literature.

\section{Introduction}

Malignant peripheral nerve sheath tumor (MPNST) is a rare soft tissue malignancy generally encountered during adulthood and only $10-20 \%$ is found in children and adolescents [1-4]. Many cases of this tumor arise in the patients with neurofibromatosis type 1 (NF1) with a poor outcome $[1,3,4]$. Although MPNST can be found in any part of the body including extremities, head and neck, trunk, or retroperitoneum $[1,2]$, intrathoracic MPNST with or without NF1 is uncommon, only with several reported adult cases [515]. In children or adolescents, only a few cases have been reported up to date [16-20].

Here, we report our experience in a rare case of huge intrathoracic MPNST in a 16-year-old adolescent with NF1. A review of the pertinent literature is included.

\section{Case Presentation}

A 16-year-old girl visited our hospital because of progressive chest discomfort and respiratory difficulties that started a month prior to the visit. Physical examination performed during her visit revealed multiple café-au-lait spots and cutaneous neurofibromas indicating NF1 in the patient as well as in the patient's mother. Her height and weight were $153 \mathrm{~cm}$ (5-10 percentile) and $48.4 \mathrm{~kg}$ (10-25 percentile), respectively. Her Tanner stage was 4 . Her blood pressure, pulse rate, respiratory rate, and body temperature were $136 / 66 \mathrm{mmHg}, 114 / \mathrm{min}, 24 / \mathrm{min}$, and $36.5^{\circ} \mathrm{C}$, respectively. Her chest radiography and chest computed tomography (CT) images revealed a large mass occupying the right thoracic cavity with multiple pleural nodules suggesting metastasis (Figures 1(a) and 1(b)). Scoliosis was associated with the tumor and multiple neurofibromas on magnetic resonance imaging of the whole spine (Figure 1(c)). The findings were suggestive of highly malignant tumor on positron emission tomography (PET)/CT imaging (Figure $1(\mathrm{~d})$ ) with a maximum standardized uptake value $\left(\mathrm{SUV}_{\max }\right)$ of 6.9. Her NF1 gene mutation analysis revealed no known overt mutation except silent mutation in C369G without change of amino acids.

She underwent immediate surgery (grossly total resection of the tumor and chest wall reconstruction with patch graft) without diagnostic biopsy because total resection of the tumor seemed feasible. Intraoperatively, the tumor was encapsulated and showed adhesion to pleura with somewhat 


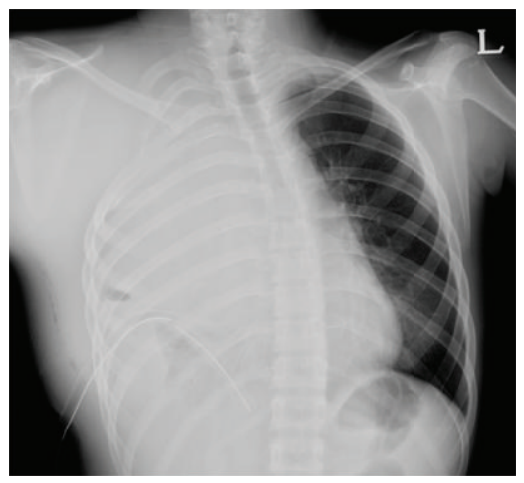

(a)

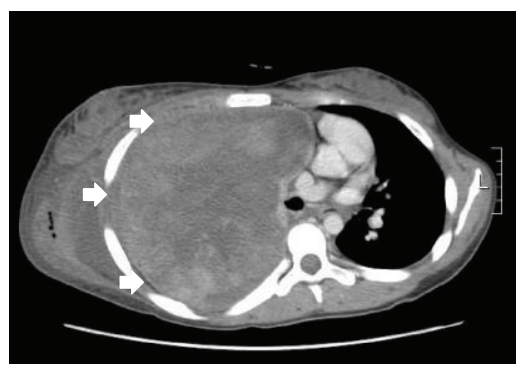

(b)

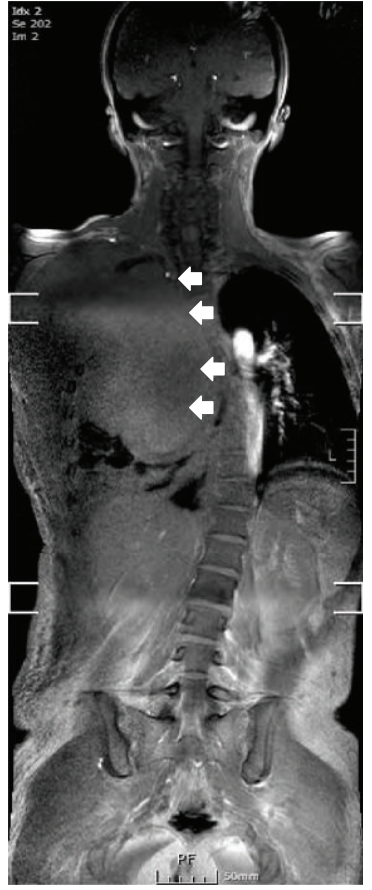

(c)

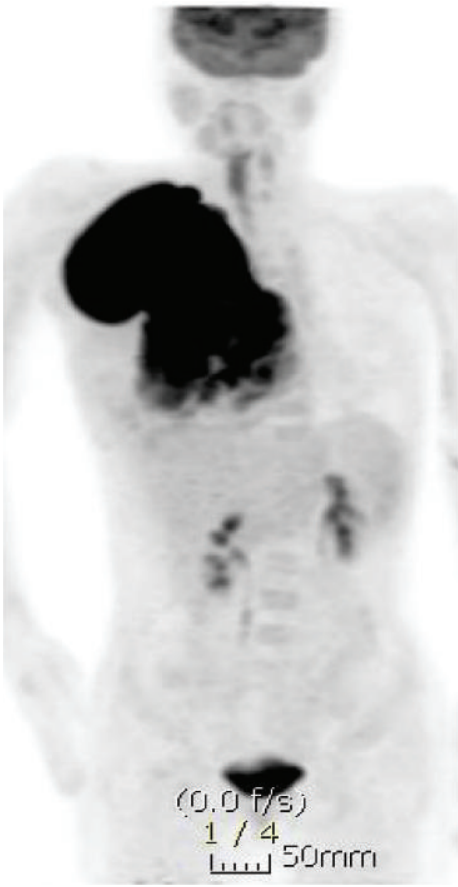

(d)

FIGURE 1: The patient's chest radiography shows a large thoracic mass with total collapse of right lung (a). Her chest CT scan (b) and spine MR imaging (c) also show a large intrathoracic hyperdense mass (white arrows) in the right chest, resulting in prominent scoliosis and cardiac deviation. Her ${ }^{18}$ F-FDG PET/CT scan showed highly increased FDG uptake $\left(S_{U} V_{\max }=6.9\right)$ in this mass, suggesting highly malignant tumor (d).

of effusion and incomplete fissure. Brachial plexus, vagus nerve, and phrenic nerve were functionally saved without tumor involvement. Macroscopically, the resected tumor was $22 \times 17 \times 9 \mathrm{~cm}$ in size (Figure 2(a)). Pulmonary metastasis was not detected in intraoperative findings. Microscopic features of the tumor showed many spindle-shaped cells with pale, eosinophilic cytoplasm (Figure 2(b)). Immunohistochemically, tumor cells expressed neuron-specific enolase (Figure 2(c)) and CD68 (focal) but not desmin, CD34, and smooth muscle actin, which were consistent with a diagnosis of MPNST.

After the surgery, she received 6,600 cGy of tomotherapy to the right whole lung field and pleura. Subsequently, she received 6 courses of chemotherapy consisting of vincristine, doxorubicin, and cyclophosphamide, alternating with ifosfamide and etoposide (VDC/IE) [21]. However, her residual pleural metastasis showed no definite response to adjuvant therapies and showed rapid progression with newly appearing pulmonary nodules. She developed a large amount of malignant pericardial effusion associated with enlarged pulmonary metastases after the sixth course of chemotherapy. Despite supportive measures including pericardiocentesis, she died of progressive disease at 9 months after the surgery.

\section{Discussion}

NF1, formerly called von Recklinghausen disease, is an autosomal dominant neurocutaneous disorder, with an estimated incidence of 1 in 3000 births $[4,16]$. NF1 is commonly associated with higher incidence in many kinds of benign or malignant tumors, such as optic pathway glioma, chronic myeloid leukemia, or pheochromocytoma, neurofibroma, and MPNST [22], and is related to mutations in NF1 gene located in chromosome 17q11.2. Because NF1 gene product named neurofibromin acts as a negative regulator in Ras signal transduction pathway, mutation in NF1 gene is related to tumor development and results in malignancies [22, 23].

MPNST is a rare soft tissue malignancy comprising about $5-10 \%$ of all soft tissue sarcomas [1-4]. Its incidence in healthy people is approximately $0.001 \%$, but its incidence is much higher in NF1 patients (2 5\%) [3,4]. Inversely, MPNST is one of the most common malignancies in NF1 patients and 50\% of MPNST arises in NF1 patients [1-3]. MPNST can be found in any part of the body, including extremities (40\%), trunk (22\%), head and neck (20\%), and retroperitoneal visceral area $(15 \%)[1,2]$. However, intrathoracic or mediastinal MPNST is very rare. Although 14 cases of intrathoracic MPNST with (8) or without (6) NF1 were reported previously in Englishlanguage literature, most of them were in adult patients with a median age of 40 (54.5 without and 39.5 with NF1) years [5-15] and only four cases with NF1 (including one with angiosarcoma component) and two without NF1 of children or adolescents have been reported (Table 1) [1620]. We were unable to find differences between the patients with and without NF1 because of limited number of the patients. Despite its rarity, intrathoracic MPNSTs in patients 


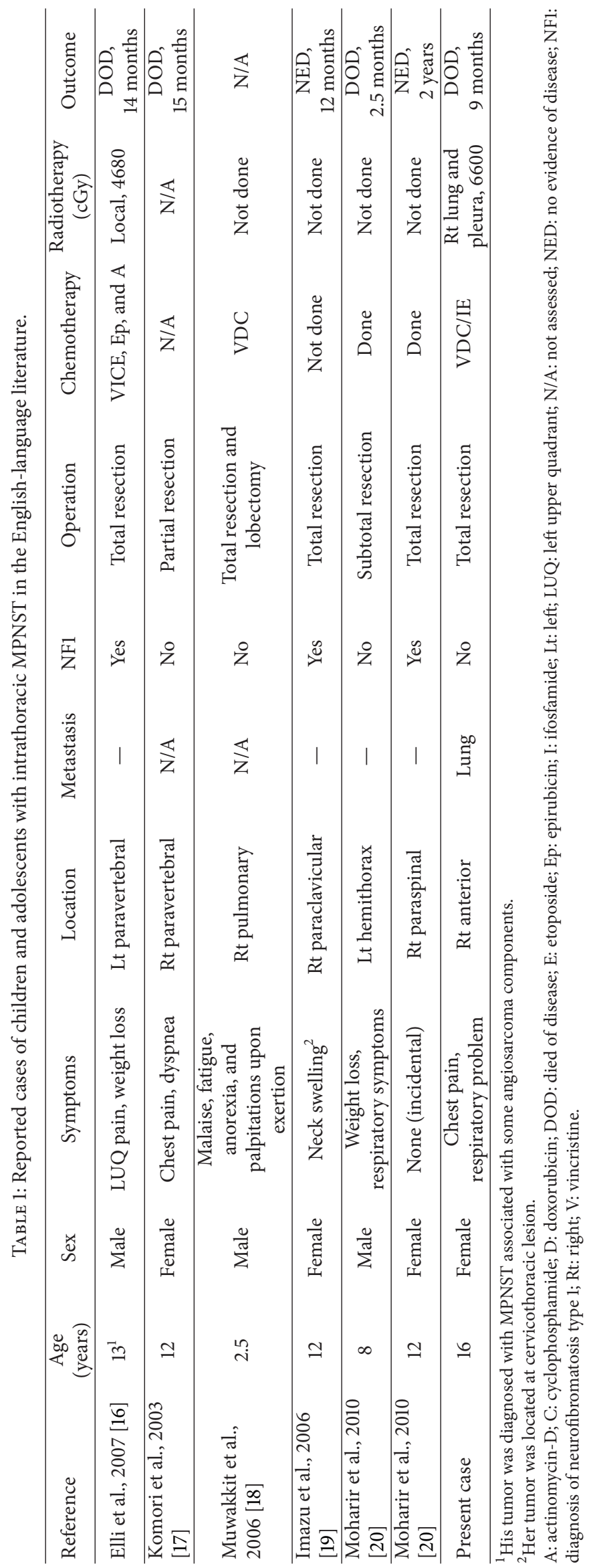




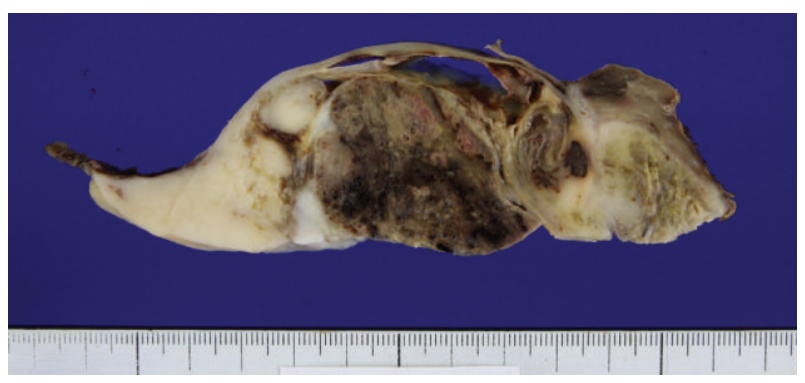

(a)

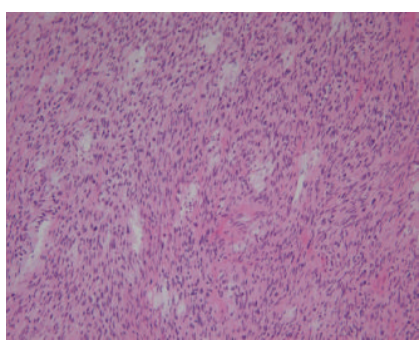

(b)

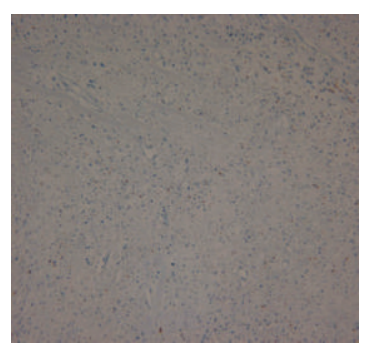

(c)
FIGURE 2: Macroscopic appearance of the cut surface of the resected tumor shows some hemorrhage and necrosis in the center. It was $22 \times 17 \times 9 \mathrm{~cm}$ in size (a). Microscopically, the tumor is composed of homogenous spindle cells (hematoxylin and eosin, $\times 100$ ) (b). Immunohistochemical stains showing positivity to neuron-specific enolase (c), consistent with MPNST.

with NF1 are thought to be originated from the plexiform neurofibromas (PNs) of thoracic nerves or vagus nerve [13]. Four (including one with angiosarcoma component) of them with NF1 had no metastasis at diagnosis, and only two survived longer than 12 months. Our patient had a tumor huge in size compared to those of previous reports with multiple pleural nodules suggesting metastasis at diagnosis, which indicates delayed diagnosis despite her NF1 stigmata. It is well known that MPNST associated with NF1 shows a poor response to chemotherapy or radiotherapy and the prognosis with residual tumor or metastasis is dismal $[1,2,18]$. Although doxorubicin/ifosfamide- (AI-) based chemotherapy is known to be somewhat effective for adult MPNST and some pediatric cases $[4,23,24]$, there is no known standard chemotherapy regimen for treatment of MPNST. Since MPNST with NF1 usually shows poor response to conventional chemotherapy we used 5-drug chemotherapy to treat our patient because VDC/IE regimen for the Ewing sarcoma or rhabdomyosarcoma may show a better response to MPNST than AI-based regimen [4, 21, 23, 24]. However, our patient also showed no response of tumor to adjuvant therapies and died of disease progression.

Wide excision of the tumor is one of the most important prognostic factors in MPNST [2]. Because some of the large intrathoracic malignant tumors cannot be removed completely due to adjacent critical vital organs, early detection of intrathoracic MPNST is very important for longterm survival of the patients with NF1 [20]. Although some assessment guidelines for children with NF1 including annual clinical evaluation of spine were suggested [25], no specific evaluation strategy for surveillance of MPNST in the patients with NF1 is known $[20,22]$. Because MPNST in NF1 patient is usually transformed from PNs and they have similar initial symptoms and signs, it is very important to distinguish small MPNST from PN for early detection of MPNST [20]. Recently, some reports suggest that ${ }^{18}$ FDG-PET/CT (with various cutoff $\mathrm{SUV}_{\max }$ of 2.5 3.5) can be a useful diagnostic tool for early detection and good prognosis of MPNST in NF1 patients [20, 26, 27]. However, considering no solid cutoff value of $\mathrm{SUV}_{\max }$ and risk of radiation exposure after ${ }^{18} \mathrm{FDG}$-PET/CT, further investigation for adequate schedule or strategy using PET/CT for early detection and rapid intervention of MPNST in NF1 should be warranted.

The authors report a rare case of huge intrathoracic MPNST in a 16-year-old girl with NF1. Considering its location and poor prognosis without complete resection, a screening strategy for early detection and intervention of MPNST in NF1 should be investigated.

\section{Conflict of Interests}

The authors declare that there is no conflict of interests regarding the publication of this paper.

\section{References}

[1] S. R. Grobmyer, J. D. Reith, A. Shahlaee, C. H. Bush, and S. N. Hochwald, "Malignant peripheral nerve sheath tumor: molecular pathogenesis and current management considerations," Journal of Surgical Oncology, vol. 97, no. 4, pp. 340-349, 2008.

[2] M. Carli, A. Ferrari, A. Mattke et al., "Pediatric malignant peripheral nerve sheath tumor: the Italian and German Soft Tissue Sarcoma Cooperative Group," Journal of Clinical Oncology, vol. 23, no. 33, pp. 8422-8430, 2005.

[3] H. A. Demir, A. Varan, B. Yalçin, C. Akyüz, T. Kutluk, and M. Büyükpamukçu, "Malignant peripheral nerve sheath tumors in childhood: 13 cases from a single center," Journal of Pediatric Hematology/Oncology, vol. 34, no. 3, pp. 204-207, 2012.

[4] A. Ferrari, G. Bisogno, and M. Carli, "Management of childhood malignant peripheral nerve sheath tumor," Pediatric Drugs, vol. 9, no. 4, pp. 239-248, 2007.

[5] R.-S. Lai, S.-L. Lin, S.-S. Hsu, and M.-T. Wu, "Intrathoracic paraspinal malignant peripheral nerve sheath tumor," Journal of the Chinese Medical Association, vol. 69, no. 1, pp. 37-41, 2006.

[6] B. H. Chao, K. A. Stogner-Underwood, J. Kiev, and T. J. Smith, "Intrathoracic malignant peripheral nerve sheath tumor in neurofibromatosis 1," Journal of Clinical Oncology, vol. 26, no. 13, pp. 2216-2218, 2008.

[7] R. Kawachi, H. Takei, G. Furuyashiki, Y. Koshi-Ishi, and T. Goya, "A malignant peripheral nerve sheath tumor of the mediastinum in a patient with neurofibromatosis type 1: report of a case," Surgery Today, vol. 38, no. 10, pp. 945-947, 2008.

[8] J. Shimizu, Y. Arano, T. Murata et al., "A case of intrathoracic giant malignant peripheral nerve sheath tumor in neurofibromatosis type i (von recklinghausen's disease)," Annals of Thoracic and Cardiovascular Surgery, vol. 14, no. 1, pp. 42-47, 2008.

[9] V. Kolarov, J. Stanić, Z. Eri, B. Zvezdin, M. Kojičić, and S. Hromis, "Intrathoracic malignant peripheral nerve sheath 
tumor with poor outcome: a case report," Bosnian Journal of Basic Medical Sciences, vol. 10, no. 4, pp. 328-330, 2010.

[10] J. H. Park, K. H. Choi, H. B. Lee, Y. K. Rhee, Y. C. Lee, and M. J. Chung, "Intrathoracic malignant peripheral nerve sheath tumor in von Recklinghausen's disease," Korean Journal of Internal Medicine, vol. 16, no. 3, pp. 201-204, 2001.

[11] T. Shimoyama, K. Yoshiya, Y. Yamato, T. Koike, and K. Honma, "Long-term survival after removal of a malignant peripheral nerve sheath tumor originating in the anterior mediastinum," General Thoracic and Cardiovascular Surgery, vol. 57, no. 6, pp. 310-314, 2009.

[12] K. Asai, K. Suzuki, H. Shimota, T. Takahashi, K. Yamashita, and T. Kazui, "Malignant peripheral nerve sheath tumor of the mediastinum: a temporary aortic transection approach," Journal of Thoracic and Cardiovascular Surgery, vol. 128, no. 4, pp. 615-617, 2004.

[13] A. Abbas, H. Jones, G. T. Kingston, and A. Zurek, "Malignant peripheral nerve sheath tumour presenting as a pneumothorax," British Journal of Radiology, vol. 84, no. 1006, pp. e197-e199, 2011.

[14] M. Inoue, T. Mitsudomi, T. Osaki, T. Oyama, J. Haratake, and K. Yasumoto, "Malignant transformation of an intrathoracic neurofibroma in von Recklinghausen's disease," Scandinavian Cardiovascular Journal, vol. 32, no. 3, pp. 173-175, 1998.

[15] H. Ogino, M. Hara, M. Satake et al., "Malignant peripheral nerve sheath tumors of intrathoracic vagus nerve," Journal of Thoracic Imaging, vol. 16, no. 3, pp. 181-184, 2001.

[16] M. Elli, B. Can, M. Ceyhan et al., "Intrathoracic malignant peripheral nerve sheath tumor with angiosarcoma in a child with NF1," Tumori, vol. 93, no. 6, pp. 641-644, 2007.

[17] M. Komori, H. Yabuuchi, T. Kuroiwa, Y. Nagatoshi, Y. Ichinose, and Y. Hachitanda, "Thoracic malignant peripheral nerve sheath tumour mimicking a pleural tumour: a rare pedunculated appearance," Pediatric Radiology, vol. 33, no. 8, pp. 578581, 2003.

[18] S. A. Muwakkit, C. Rodriguez-Galindo, A. I. El Samra et al., "Primary malignant peripheral nerve sheath tumor of the lung in a young child without neurofibromatosis type 1," Pediatric Blood and Cancer, vol. 47, no. 5, pp. 636-638, 2006.

[19] M. Imazu, Y. Nakamura, H. Nakatani et al., "Cervicothoracic malignant peripheral nerve sheath tumor in a 12-year-old girl with neurofibromatosis type 1," European Journal of Pediatric Surgery, vol. 16, no. 4, pp. 285-287, 2006.

[20] M. Moharir, K. London, R. Howman-Giles, and K. North, "Utility of positron emission tomography for tumour surveillance in children with neurofibromatosis type 1," European Journal of Nuclear Medicine and Molecular Imaging, vol. 37, no. 7, pp. 13091317, 2010.

[21] C. A. S. Arndt, D. S. Hawkins, W. H. Meyer, S. F. Sencer, J. P. Neglia, and J. R. Anderson, "Comparison of results of a pilot study of alternating vincristine/ doxorubicin/cyclophosphamide and etoposide/ifosfamide with IRS-IV in intermediate risk rhabdomyosarcoma: a report from the children's oncology group," Pediatric Blood and Cancer, vol. 50, no. 1, pp. 33-36, 2008.

[22] B. R. Korf, "Malignancy in neurofibromatosis type 1," Oncologist, vol. 5, no. 6, pp. 477-485, 2000.

[23] J. R. Kroep, M. Ouali, H. Gelderblom et al., "First-line chemotherapy for malignant peripheral nerve sheath tumor (MPNST) versus other histological soft tissue sarcoma subtypes and as a prognostic factor for MPNST: an EORTC Soft Tissue and Bone Sarcoma Group study," Annals of Oncology, vol. 22, no. 1, pp. 207-214, 2011.

[24] L. Granowetter, R. Womer, M. Devidas et al., "Dose-intensified compared with standard chemotherapy for nonmetastatic ewing sarcoma family of tumors: a children's oncology group study," Journal of Clinical Oncology, vol. 27, no. 15, pp. 25362541, 2009.

[25] R. E. Ferner, S. M. Huson, N. Thomas et al., "Guidelines for the diagnosis and management of individuals with neurofibromatosis," Journal of Medical Genetics, vol. 44, no. 2, pp. 81-88, 2007.

[26] V. S. Warbey, R. E. Ferner, J. T. Dunn, E. Calonje, and M. J. O'Doherty, "FDG PET/CT in the diagnosis of malignant peripheral nerve sheath tumours in neurofibromatosis type-1," European Journal of Nuclear Medicine and Molecular Imaging, vol. 36, no. 5, pp. 754-757, 2009.

[27] W. Brenner, R. E. Friedrich, K. A. Gawad et al., "Prognostic relevance of FDG PET in patients with neurofibromatosis type1 and malignant peripheral nerve sheath tumours," European Journal of Nuclear Medicine and Molecular Imaging, vol. 33, no. 4, pp. 428-432, 2006. 


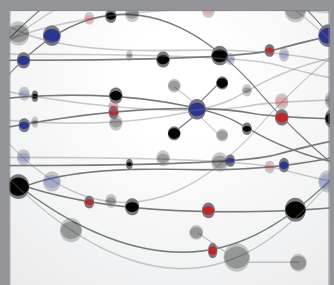

The Scientific World Journal
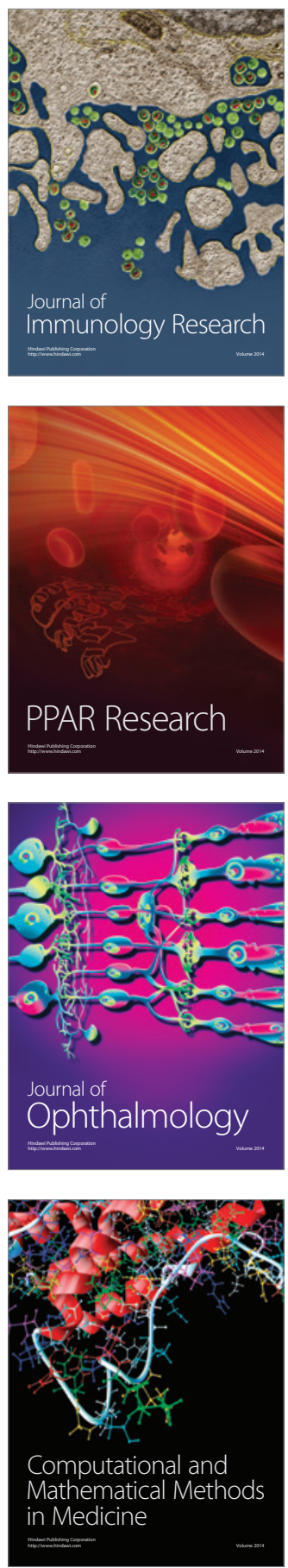

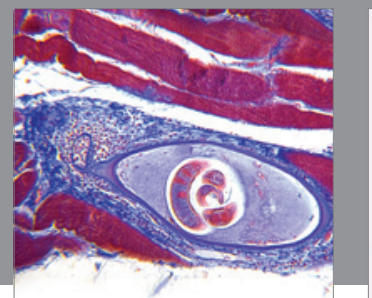

Gastroenterology

Research and Practice
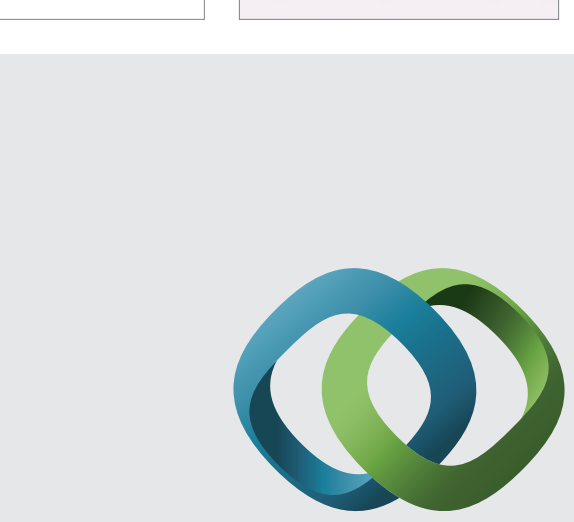

\section{Hindawi}

Submit your manuscripts at

http://www.hindawi.com
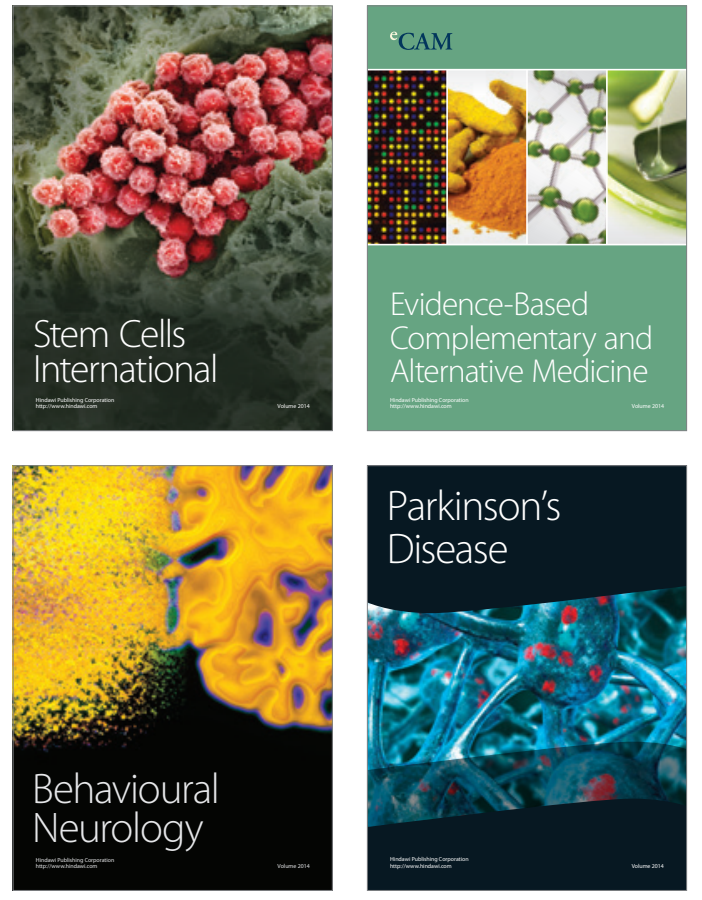
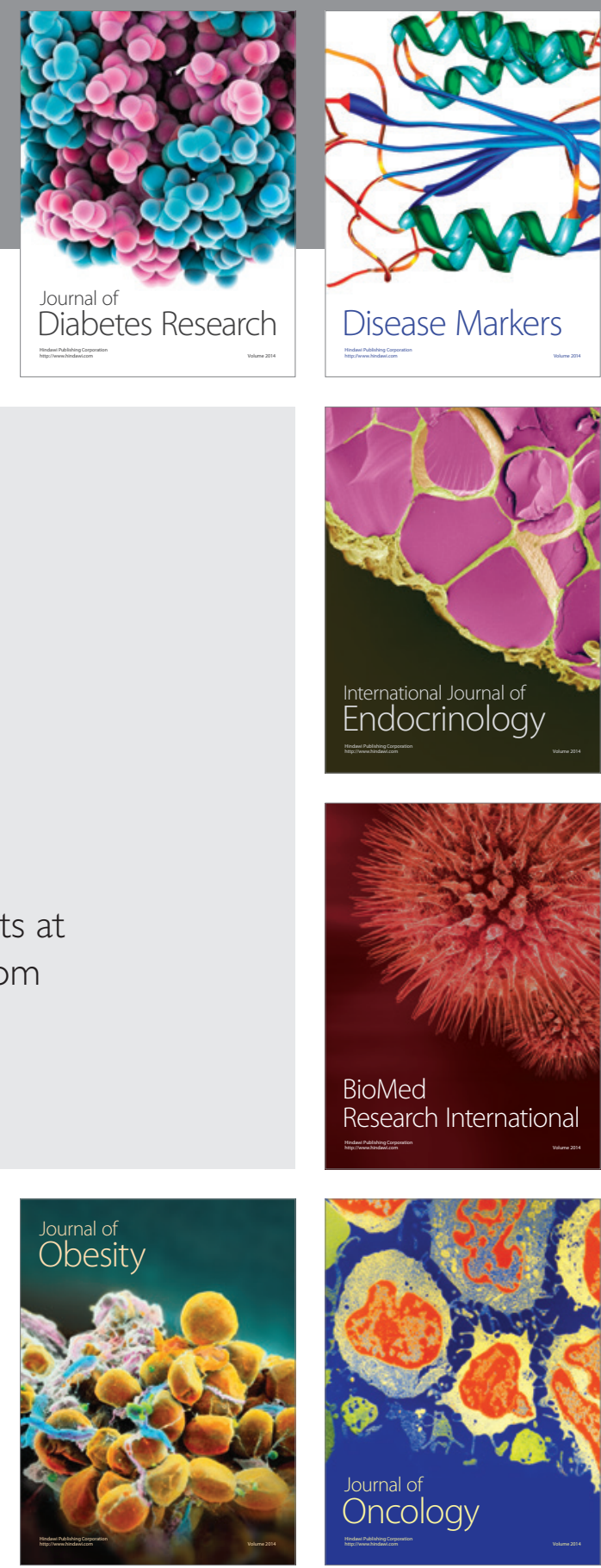

Disease Markers
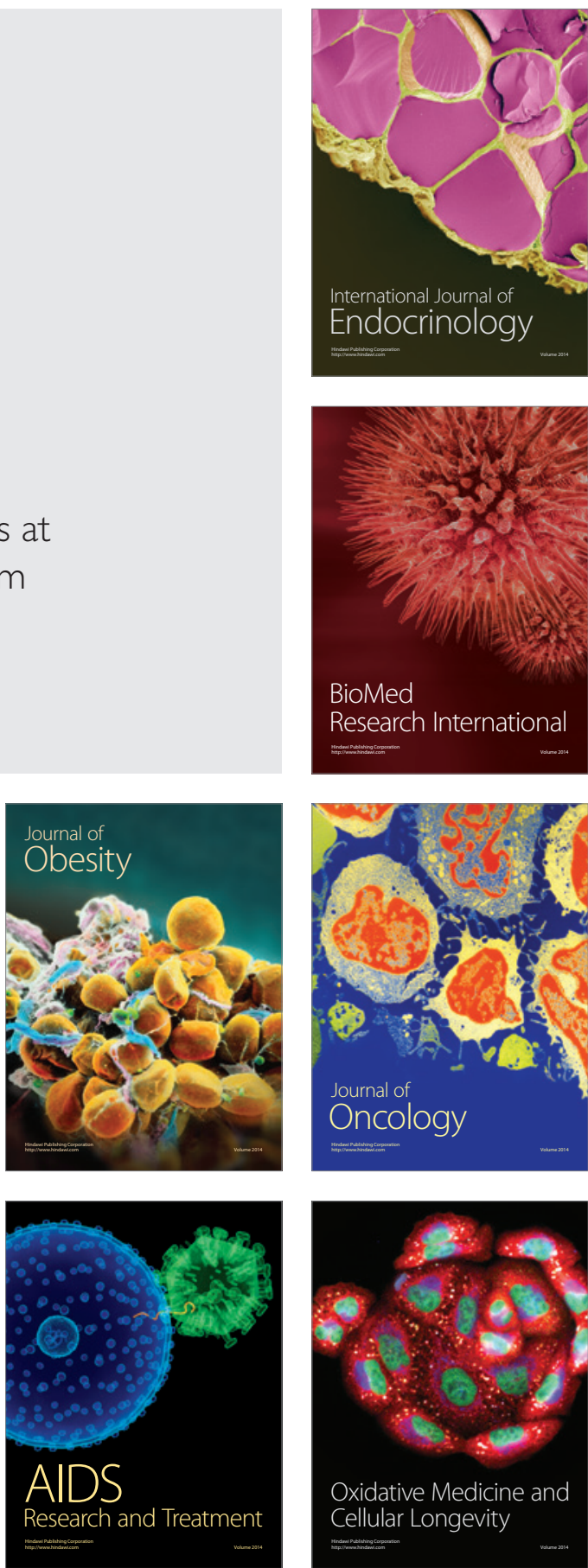